\title{
Consolidated Numerical Simulation of Multiple Flows with Heat Transfer using Virtual Flux Method*
}

\author{
Hidetoshi NISHIDA $^{* *}$ and Hiroyuki SASAOKA ${ }^{* *}$ \\ ${ }^{* *}$ Department of Mechanical and System Engineering, Graduate School of Science and Technology, \\ Kyoto Institute of Technology, \\ Matsugasaki, Sakyo-ku, Kyoto 606-8585, Japan \\ E-mail: nishida@kit.ac.jp
}

\begin{abstract}
In this paper, the Cartesian grid approach with virtual flux method based on the usual incompressible Navier-Stokes equations is proposed for simulating the incompressible multiple flows with heat transfer. In this approach, the fluxes on the closest grid points to virtual boundary are estimated to satisfy the velocity, pressure and temperature conditions on the virtual boundary. First, the present method is validated in flows around a circular cylinder and a pair of circular cylinders in transverse arrangement. As a result, this approach gives the good flow fields quantitatively in comparison with the reference results. Then, the method is applied to the consolidated simulations of multiple flows with heat transfer between heated and cooling flows. Therefore, it is concluded that the present approach is very fruitful for the consolidated simulation of incompressible complicated multiple flows with heat transfer.
\end{abstract}

Key words: CFD, Consolidated Numerical Simulation, Virtual Flux Method, Incompressible Multiple Flows

\section{Introduction}

By downsizing and complication of the recent engineering equipment, the flow analysis with it also becomes more complicated. In the practical complicated flow, it is necessary to predict the multiple flows with heat transfer. There are two difficulties of this complicated flow analysis. One is the grid generation and another is the establishment of approach for consolidated numerical simulation of multiple flows. In the complicated flow analysis, the boundary fitted coordinates (BFC) is usually adopted. This BFC approach has the high adaptability to the boundary configuration. However, in the complicated flow geometry, it is difficult to generate the computational grid. In some cases, the time spent for the generation of computational grid is lengthened further than the computational time for practical flow analysis. Therefore, in recent years, the Cartesian grid approach is highlighted again for the numerical flow analysis.

In the Cartesian grid approaches, especially, the immersed boundary method ${ }^{(1)-(4)}$ is applied to many simulations of incompressible flow. In order to satisfy the velocity conditions on the boundary points, the immersed boundary method fundamentally requires the external forcing term added to the momentum equations. Another approach named the virtual flux method is proposed recently by Tanno et al. ${ }^{(5),(6)}$. In this virtual flux method, the fluxes of the governing equations on the closest grid point to the virtual boundary are estimated to satisfy the velocity, pressure, and temperature conditions on the virtual 
boundary. By using this virtual flux method, the multiple flows with heat transfer can be simulated consolidatively on the Cartesian grid system. However, the original virtual flux method is formulated in the pseudo-compressibility equations in which the velocity and pressure at next time step are computed by the relaxation scheme simultaneously. Then, the adaptability to the usual incompressible Navier-Stokes equations without the velocity relaxation is not validated. Also, the homogeneous Neumann boundary condition for the pressure, $\partial p / \partial n=0$, is used in the original method. For application to many practical flow simulations, it is very important to validate the virtual flux method in the usual incompressible Navier-Stokes equations with the usual Neumann boundary condition for the pressure.

In this paper, the virtual flux method formulated in the pseudo-compressible Navier-Stokes equations is reformulated in the usual incompressible Navier-Stokes equations with the usual pressure boundary condition. First, the present virtual flux method is validated in the flows around a circular cylinder and a pair of circular cylinder. Secondly, this approach is applied to the consolidated numerical simulation of heated flows in pipes and cooling flow around pipes in order to confirm the adaptability of 3D multiple flows with heat transfer. Finally, the counter-flow heat exchanger models are considered.

\section{Virtual Flux Method}

\subsection{Governing equations}

The incompressible viscous flow with heat transfer is governed by the continuity equation, the incompressible Navier-Stokes equations, and the energy equation. These equations can be written in nondimensional form by

$$
\begin{aligned}
& \frac{\partial u_{i}}{\partial x_{i}}=0, \\
& \frac{\partial u_{i}}{\partial t}+u_{j} \frac{\partial u_{i}}{\partial x_{j}}=-\frac{\partial p}{\partial x_{i}}+\frac{1}{R e} \frac{\partial^{2} u_{i}}{\partial x_{j} \partial x_{j}}, \\
& \frac{\partial T}{\partial t}+u_{j} \frac{\partial T}{\partial x_{j}}=\frac{1}{\operatorname{Pr} \operatorname{Re}} \frac{\partial^{2} T}{\partial x_{j} \partial x_{j}},
\end{aligned}
$$

where $u_{i}, p$, and $T$ denote the velocity component, the pressure, and the temperature. $R e$ $(=U L / v)$ denotes the Reynolds number and $\operatorname{Pr}(=\alpha / v)$ is the Prandtl number. $U, L, \alpha$, and $v$ are the reference velocity, the reference length, the thermal diffusivity, and the kinematic viscosity, respectively.

\subsection{Computational techniques}

The incompressible Navier-Stokes equations (2) and the energy equation (3) are solved by the second order finite difference method on the collocated grid arrangement. The convective, diffusion, and pressure terms except the closest grid points to the virtual boundary are discretized by the second order fully conservative finite difference method ${ }^{(7)}$, and the usual second order centered finite difference method. For the time integration, the fractional step approach ${ }^{(8)}$ based on the forward Euler scheme is applied.

$$
u_{i}^{*}=u_{i}^{n}+\Delta t F_{i}, u_{i}^{n+1}=u_{i}^{*}-\Delta t \frac{\partial p}{\partial x_{i}},
$$

where $u_{i}{ }^{*}$ denotes the fractional step velocity and $F_{i}$ is the convective and diffusion terms. The resulting pressure equation, $\nabla^{2} p^{n}=\nabla \cdot u_{i}{ }^{*} / \Delta t$, is solved by the SOR method. The 
convergence criterion of the pressure equation is set as $p_{L 2}<1.0 \times 10^{-6}$, where $p_{L 2}$ is the L-2 residual of the pressure.

\subsection{Virtual flux method}

The virtual flux method can calculate the flows around the arbitrary bodies and in the arbitrary geometry on the Cartesian grid. This method has the advantage in which the flux on the closest grid points to the virtual boundary is changed only, so that the implementation is very easy. In order to explain the estimation of virtual flux, we consider 2D case, but the extension to 3D is straightforward. Figure 1 shows the schematic view of the virtual flux method. The solid circle denotes the grid points on the Cartesian coordinates. The virtual boundary is displayed by the dashed line. The circular and triangular symbols denote the virtual boundary points in the $x$ and $y$ directions, respectively. Two fluid regions, i.e., region A and region B, are separated by these virtual boundary points.

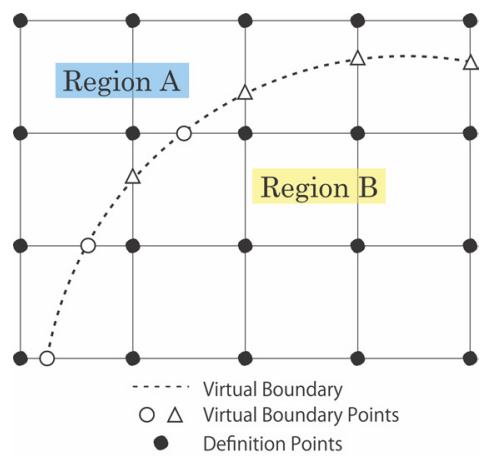

Fig. 1 Schematic view of virtual flux method.

The physical quantity vector is defined by $\boldsymbol{q}=\left(u_{i}, p, T\right)^{T}$. As shown in Fig.2, the virtual boundary point is arranged between the grid points $(i, j)$ and $(i+1, j)$. The physical quantities on the grid point, $\boldsymbol{q}_{i, j}$ and $\boldsymbol{q}_{i+1, j}$, and ones on the virtual boundary, $\boldsymbol{q}_{v b}$, are known. Usually, the physical quantities on $(i-1, j),(i, j)$, and $(i+1, j)$ are used for discretizing the spatial derivatives of momentum equations. In the present virtual flux method, the physical quantities on $(i+1, j), \boldsymbol{q}_{i+1, j}$, is modified to satisfy the conditions on the virtual boundary, $\boldsymbol{q}_{v b}$. The modified physical quantities $\boldsymbol{q}^{\boldsymbol{*}_{i+1, j}}$ is estimated by the linear interpolation. As a result, the spatial derivatives are discretized by using $\boldsymbol{q}_{i-1, j}, \boldsymbol{q}_{i, j}$ and $\boldsymbol{q}^{\boldsymbol{*}_{i+1, j}}$. However, when the distance between the grid point and the virtual boundary point is small, the calculation may fail because the denominator of interpolation is close to zero. In this case, it is necessary to change the estimation of the virtual flux. The physical quantities, $\boldsymbol{q}^{*}{ }_{i+1, j}$, are estimated by using $\boldsymbol{q}_{i-1 / 2, j}$ which is defined on $(i-1 / 2, j)$ shown in Fig.3.

In order to estimate the virtual flux, it is necessary that the physical quantities on the virtual boundary point, $\boldsymbol{q}_{v b}$, are known. The velocity on the virtual boundary points is given by the specified velocity, $u_{i}=u_{v b}$, e.g., $u_{v b}=0$ for the non-slip condition. The pressure on the virtual boundary is determined by the pressure derivative obtained by the normal momentum equation, i.e., $\partial p / \partial x_{i}=F_{i}$. The temperature on the boundary is specified by $T=T_{v b}$ for the isothermal condition or $\partial T / \partial n=0$ for the adiabatic condition. For the

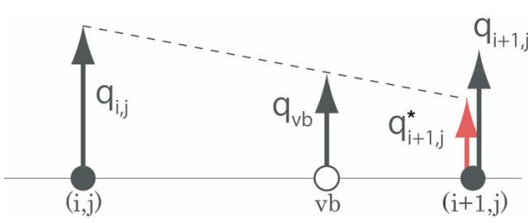

Fig. 2 Estimation of virtual flux.

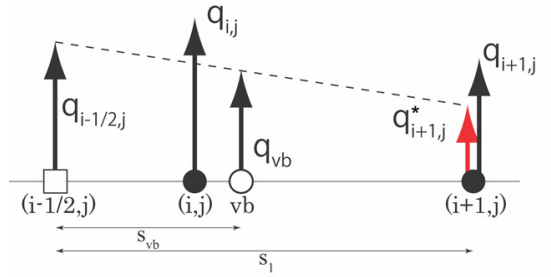

Fig. 3 Estimation for small distance case. 
Neumann conditions, $\boldsymbol{q}^{*}{ }_{i+1, j}$ is determined by its derivative. Especially, for the inner field of stationary solid media, the velocity is $u_{\text {inner }}=0$ and the pressure is determined by solving the pressure equation to satisfy the Neumann boundary condition on the boundary surface. The temperature at next time step is computed with $u_{\text {inner }}=0$, so that the heat transfer in the solid media is estimated by the parabolic partial differential equation. Also, the heat transfer between flow and solid is considered by the continuity condition of heat flux,

$$
\frac{\partial}{\partial x_{i}}\left(\frac{1}{\operatorname{Pr} R e} \frac{\partial T}{\partial x_{i}}\right)=0
$$

\section{Validation of Virtual Flux Method}

\subsection{Flow around a circular cylinder}

Figure 4 shows the computational domain and the used non-uniform grid. The non-uniform grid system with $248 \times 248$ grid points is adopted. The initial state is given by the impulsive start determined by the uniform flow with $T=0.0$. On the inflow boundary, the velocity and temperature are fixed by the uniform flow and the pressure is imposed by the linear extrapolation from the inner points. The velocity and temperature are extrapolated from the inner points and the pressure is obtained by the Sommerfeld radiation condition ${ }^{(9)}$ on the outflow boundary. On the virtual boundary, i.e., the surface of a circular cylinder, the non-slip velocity condition, $u_{v b}=0.0$, the Neumann condition obtained by $\partial p / \partial x_{i}=F_{i}$ for the pressure, and the isothermal condition, $T_{v b}=1.0$, are imposed. The Prandtl number is $\operatorname{Pr}=0.717$.
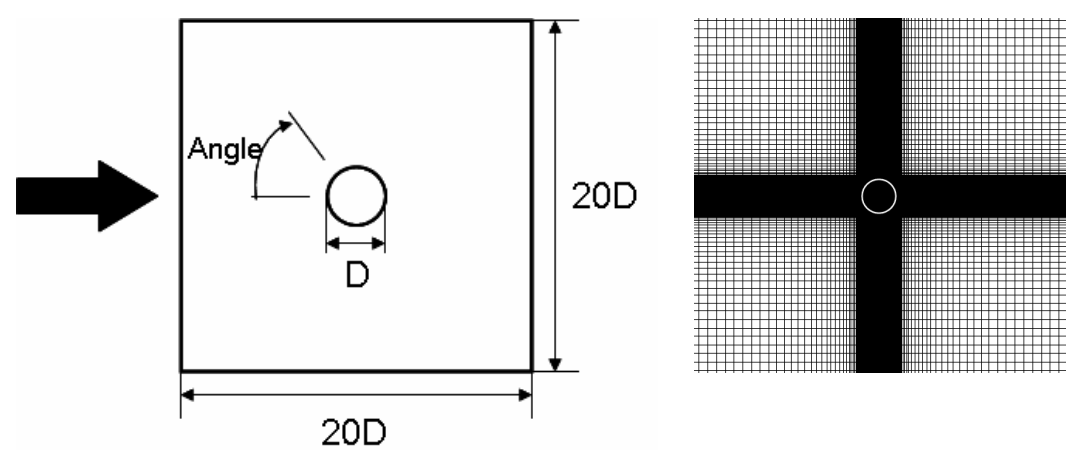

Fig. 4 Computational domain and non-uniform grid for flow around a circular cylinder.

Figure 5 shows the snap shots of the pressure, vorticity, and temperature with $R e=200$. The smooth flow field near the boundary is obtained. The vortex shedding can be observed. Table 1 shows the comparison of characteristic quantities, i.e., $C_{\text {Dmean }}, C_{\text {Lamp }}$, and St. The present solution is in good agreement with the reference ones ${ }^{(10),(11)}$.

Table 1 Comparison of characteristic quantities with $R e=200$.
\begin{tabular}{|l|c|c|c|}
\hline & $C_{\text {Dmean }}$ & $C_{\text {Lamp }}$ & $S t$ \\
\hline Present & 1.301 & 0.658 & 0.197 \\
\hline Rosenfeld $^{(10)}$ & 1.329 & 0.674 & 0.197 \\
\hline Rogers et al. $^{(11)}$ & 1.230 & 0.650 & 0.185 \\
\hline
\end{tabular}

In order to validate the result with heat transfer, the time averaged local Nusselt number with $R e=218$ is shown in Fig.7. Figure 6 shows the averaged Nusselt number with the various Reynolds number. It is confirmed that the present approach gives the appropriate thermal flow in comparison with the reference results ${ }^{(6),(12)-(14)}$. 

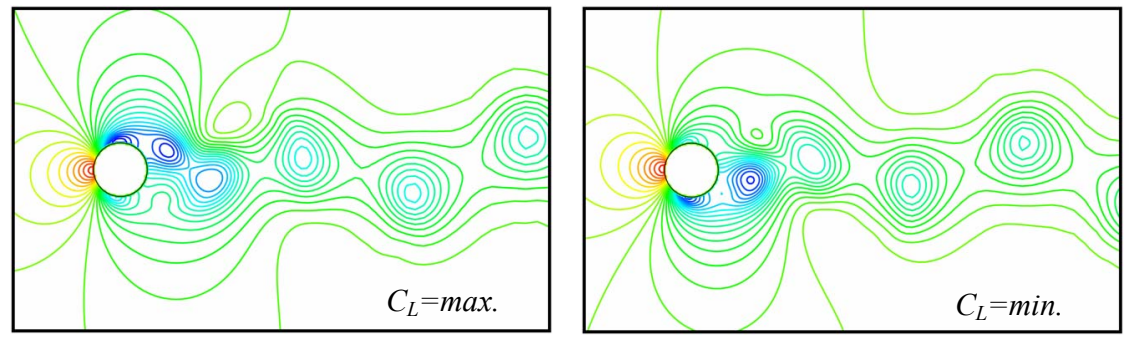

(a) Pressure contours.
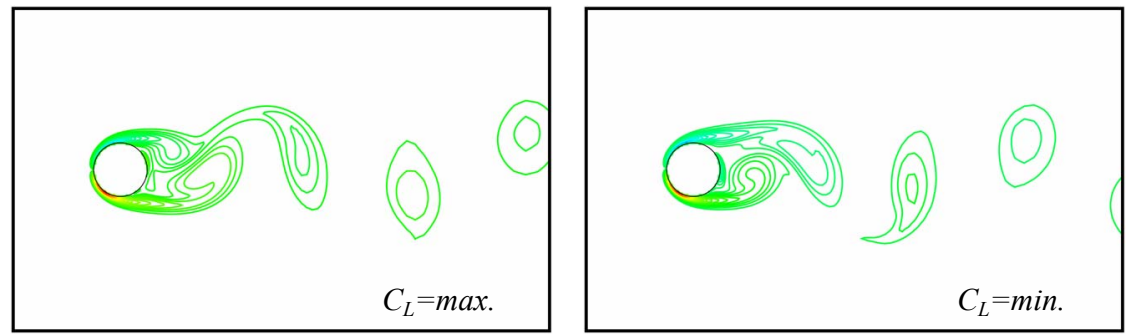

(b) Vorticity contours.
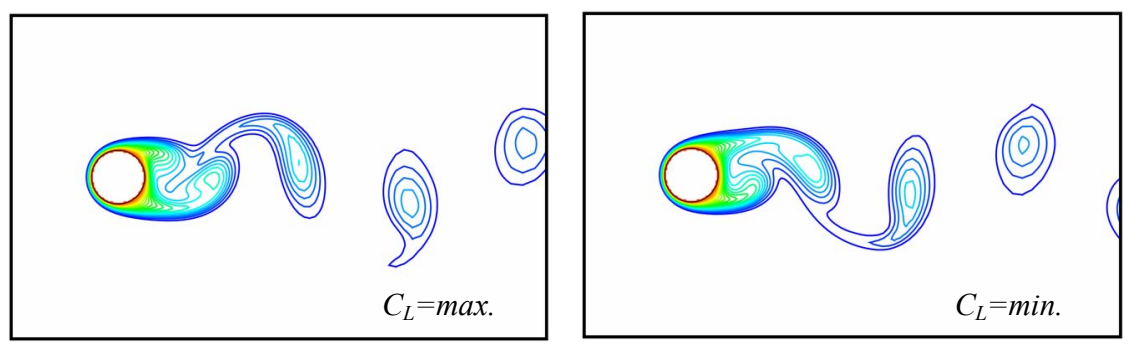

(c) Temperature contours.

Fig. 5 Snap shots of flow field with $R e=200$.

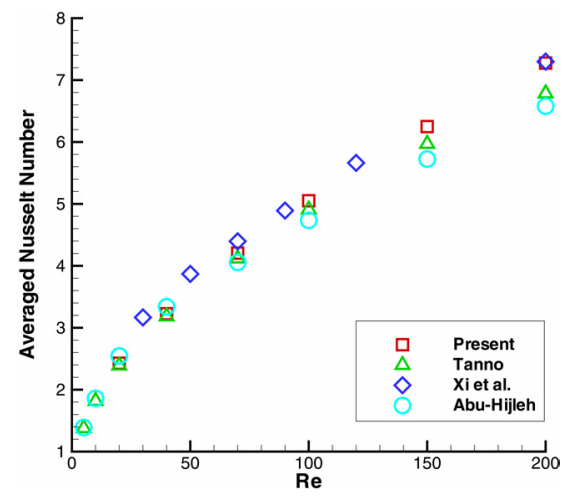

Fig. 6 Relation between Reynolds number and Nusselt number.

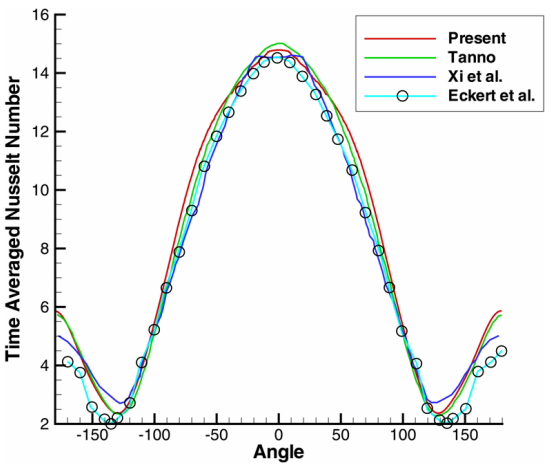

Fig. 7 Time averaged local Nusselt number distribution $(R e=218)$.

\subsection{Flow around a pair of circular cylinders}

In this section, the flow around a pair of circular cylinders in transverse arrangement is considered. Chang et al. ${ }^{(15)}$ reported that the vortex shed with antiphase in early stage of calculation, thereafter the flow shifted to the inphase motion. The computational domain is shown in Fig.8. The simulation with $620 \times 480$ grid points on non-uniform grid system which is similar to the previous case is carried out. The initial and boundary conditions, and the conditions on the virtual boundary are the same as the previous simulation.

Figure 9 shows the flow field in the antiphase and inphase motions with $R e=100$. Table 2 denotes the comparison of characteristic quantities of upper cylinder. At early stage, 


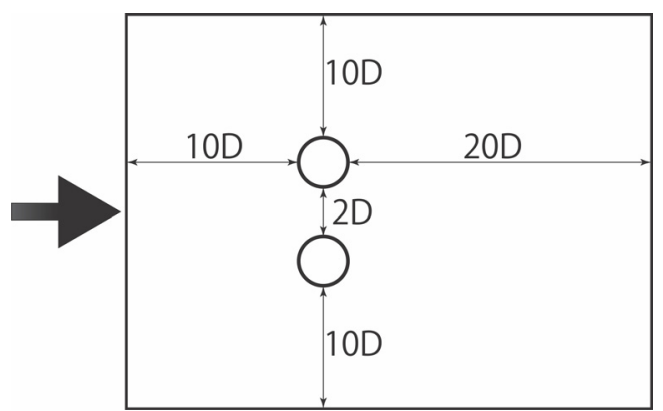

Fig. 8 Computational domain for flow around a pair of circular cylinders.
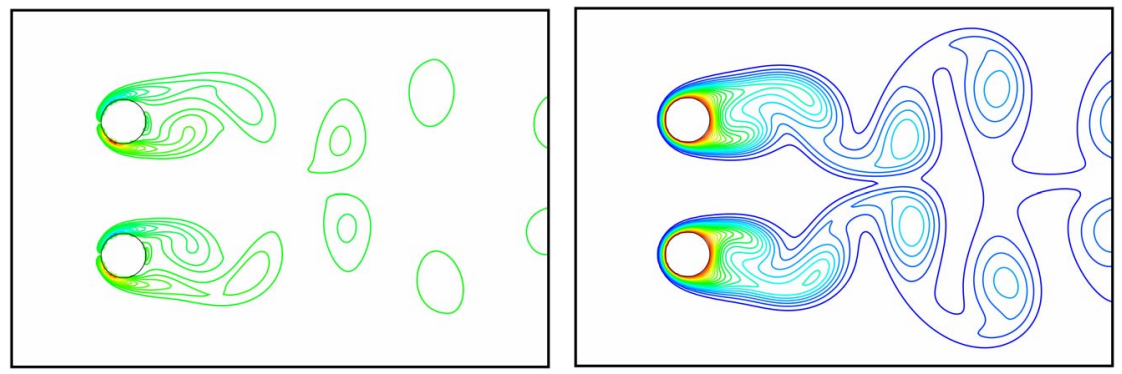

(a) Antiphase.

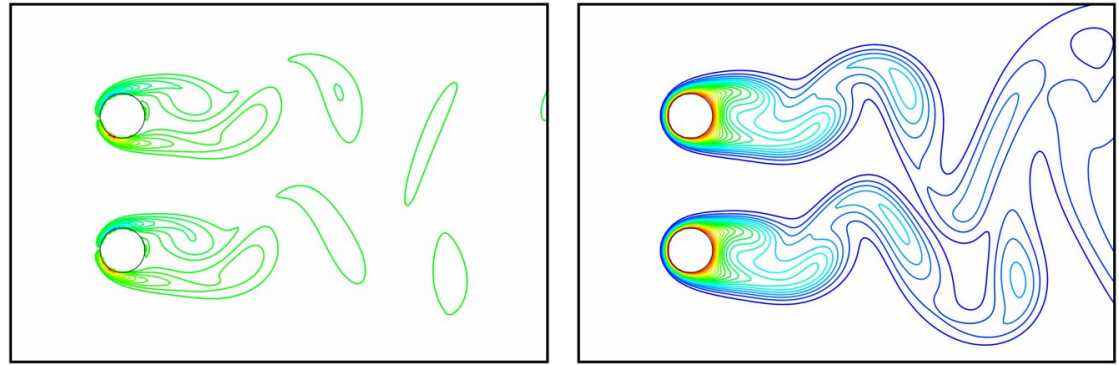

(b) Inphase.

Fig. 9 Vorticity (left) and temperature (right) contours $(R e=100)$.

Table 2 Comparison of characteristic quantities of upper cylinder with $R e=100$.

\begin{tabular}{|l|c|c|c|c|c|}
\hline \multicolumn{1}{|c|}{ Antiphase } & $C_{\text {Dmean }}$ & $C_{\text {Damp }}$ & $C_{\text {Lmean }}$ & $C_{\text {Lamp }}$ & $S t$ \\
\hline Present $^{(5)}$ & 1.439 & 0.036 & 0.115 & 0.429 & 0.184 \\
\hline Tanno $^{(15)}$ & 1.521 & 0.039 & 0.114 & 0.490 & 0.185 \\
\hline Chang et al. $^{(15)}$ & 1.525 & 0.030 & 0.104 & 0.310 & 0.180 \\
\hline \hline \multicolumn{1}{|c|}{ Inphase } & $C_{\text {Dmean }}$ & $C_{\text {Damp }}$ & $C_{\text {Lmean }}$ & $C_{\text {Lamp }}$ & $S t$ \\
\hline Present $^{\text {(5) }}$ & 1.422 & 0.030 & 0.127 & 0.261 & 0.177 \\
\hline Tanno $^{(15)}$ & 1.496 & 0.035 & 0.117 & 0.200 & 0.179 \\
\hline Chang et al. $^{(15)}$ & 1.533 & 0.040 & 0.108 & 0.310 & 0.180 \\
\hline
\end{tabular}

$t=200$, the flow displays the antiphase motion. As time goes by, the inphase motion is observed $(t=1000)$. The characteristic quantities obtained by the present approach agree with other reference solutions ${ }^{(5),(15)}$. Then, the present virtual flux method is effective for the simulation of flow around multiple bodies.

\section{Consolidated Simulation of Heated Flows in Pipes and Cooling Flow around Pipes}

\subsection{Heated flow in a pipe}

First, we consider the consolidated simulation of heated flow in a pipe and the cooling 
flow around a pipe. The thickness of pipe is $0.1 D$, where $D$ denotes the outer diameter of a pipe. The outline of multiple flows is shown Fig.10. The non-uniform grid with $204 \times 190 \times 12$ grid points is used. The impulsive start determined by the uniform flow with $T=1.0$ for the internal flow and $T=0.0$ for the external flow. For the internal heated flow, the pressure is fixed on the outflow boundary. For the external flow, the velocity and pressure are imposed by the periodic condition and the temperature is extrapolated from the inner points on the side boundaries. The other boundary conditions are the same as the previous simulation. The heat transfer between flows and pipe is estimated by the continuity condition of heat flux, Eq.(5). The Prandtl number is $P r=0.71$ for fluid and $P r=0.71$ and 0.14 for inside the pipe wall. The Reynolds number is $R e=120$.

Figure 11 shows the vorticity and temperature contours on $z=1.0 \mathrm{D}$ plane with the wall Prandtl number $P r=0.14$ and 0.71 . In both cases, the vortex shedding can be observed, but the heat transfer with $P r=0.71$ is much more than the case with $P r=0.14$. This is shown in Fig. 12 in which the temperature contours on $z=1.0 \mathrm{D}$ plane for near the pipe is displayed at $t=200$. Clearly in the high wall Prandtl number, the heat transfer from the wall to the

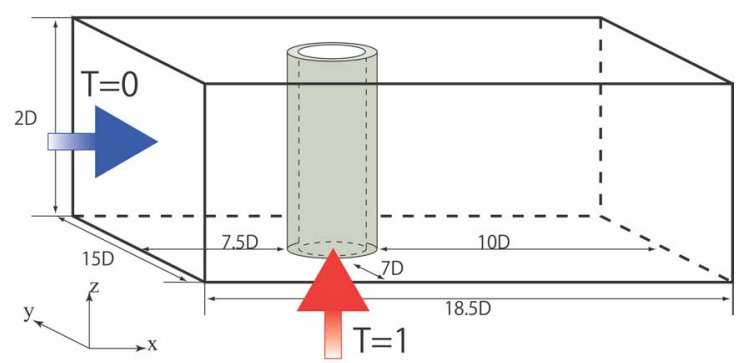

Fig. 10 Computational domain for heated flow in a pipe and cooling flow around a pipe.
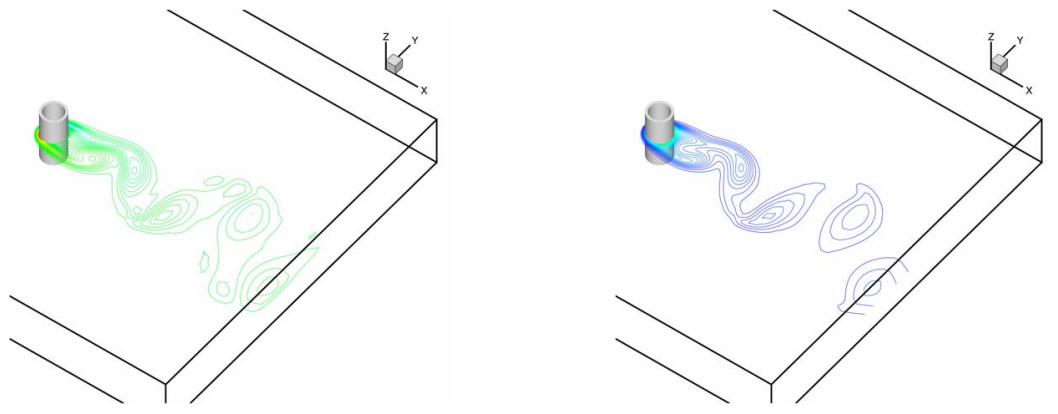

(a) $\operatorname{Pr}=0.14$.
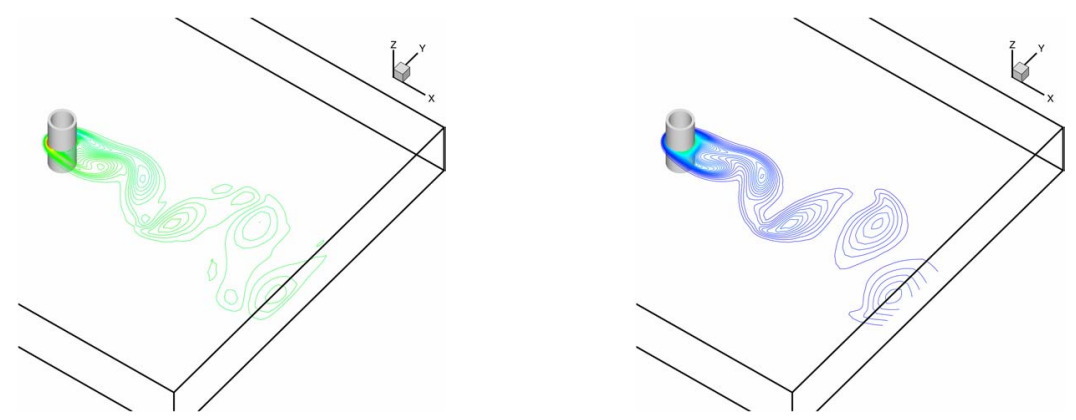

(b) $\operatorname{Pr}=0.71$.

Fig. 11 Snap shots of vorticity (left) and temperature (right) contours. 


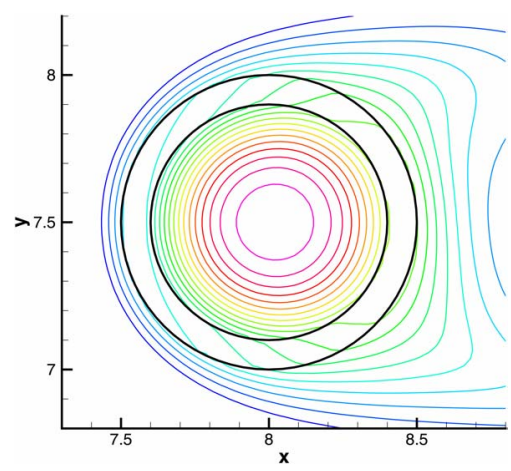

(a) $\operatorname{Pr}=0.14$.

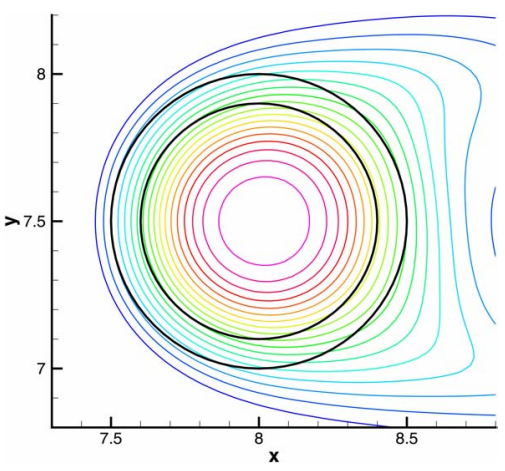

(b) $\operatorname{Pr}=0.71$.

Fig. 12 Temperature contours near a pipe $(z=1.0 D, t=200)$.

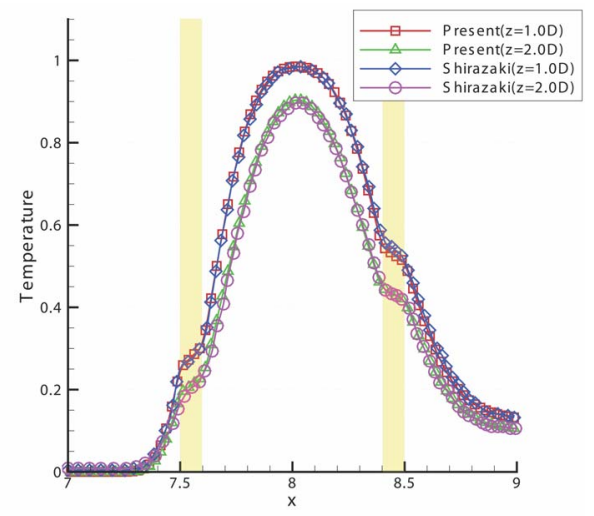

(a) $\operatorname{Pr}=0.14$.

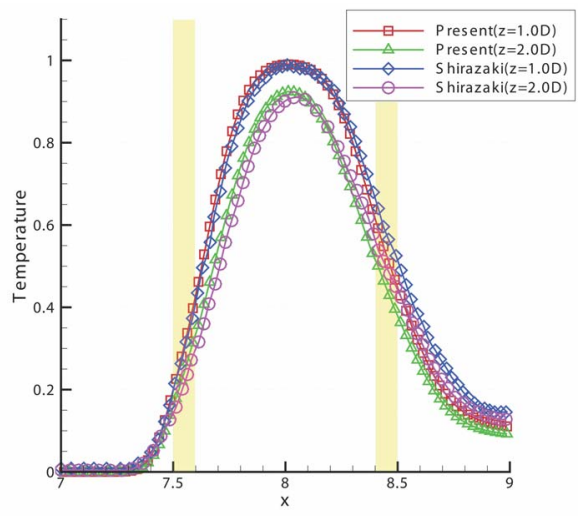

(b) $\operatorname{Pr}=0.71$.

Fig. 13 Time averaged temperature distributions on $y=7.5 \mathrm{D}$.

external flow is more active than the low wall Prandtl number. Figure 13 shows the temperature distribution on $y=7.5 \mathrm{D}$ plane. In the wall Prandtl number $\operatorname{Pr}=0.14$, the temperature refracts on the boundary between fluid and wall. In comparison with the reference solution ${ }^{(16)}$, the present approach gives the well solution.

\subsection{Heated flows in a pair of pipes}

The two pipes which is the same shape as the previous simulation are arranged in Fig.14. The distance between both centers of pipe is $2.0 D$. The grid resolution is set as the non-uniform grid with $392 \times 190 \times 12$ grid points. The Reynolds number is $R e=120$ and the Prandtl number is $P r=0.71$. The other computational conditions are the same as the case of single pipe.

Figure 15 shows the temperature contours on $z=1.0 D$ plane. In the upstream pipe, the vortex shedding does not appear, but the vortex shedding is formed in the downstream pipe. The time averaged temperature distributions on the outer surface of both pipes are shown in Fig.16. As the distance from inflow boundary of the heated flows becomes longer, the

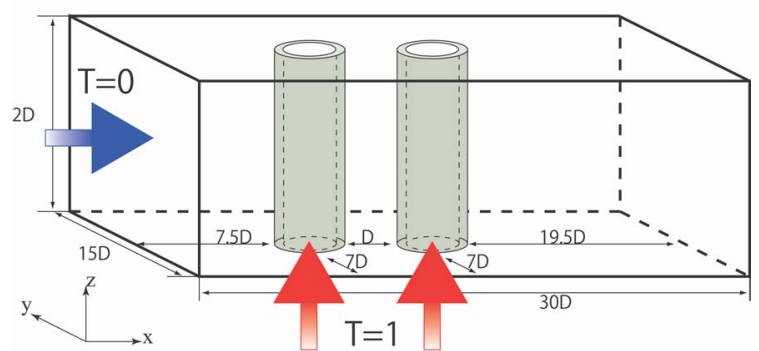

Fig. 14 Computational domain for heated flow in a pair of pipes and cooling flow around pipes. 


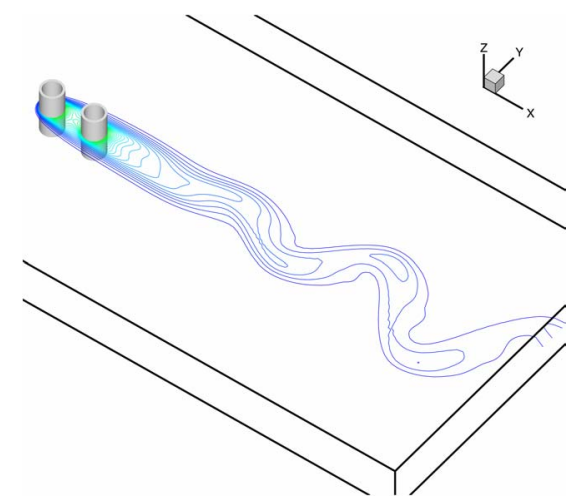

(a) Whole view.

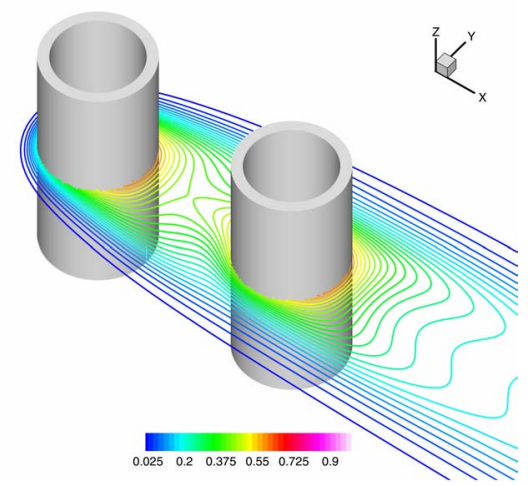

(b) Close-up view.

Fig. 15 Temperature contours on $z=1.0 D$.

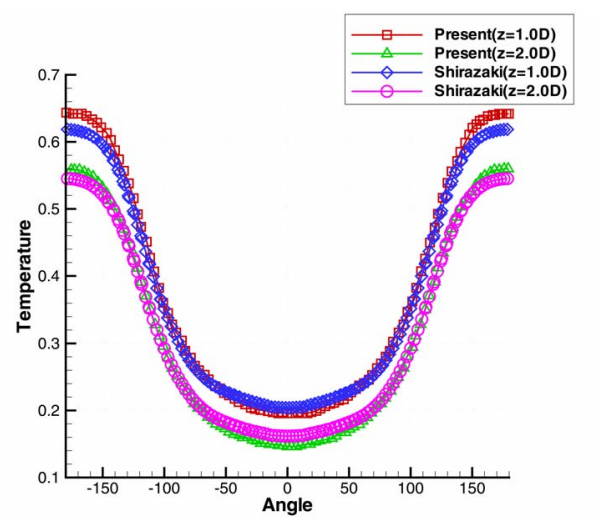

(a) Upstream.

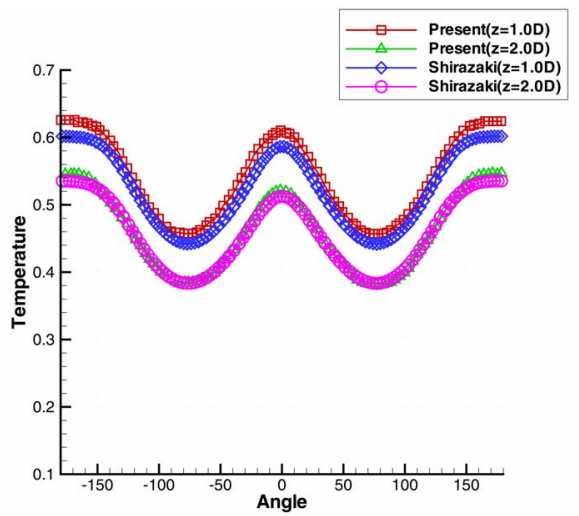

(b) Downstream.

Fig. 16 Time averaged temperature distributions of external surfaces.

temperature on the outer surface becomes lower. On the upstream pipe, the temperature reaches the lowest value at $\theta=0^{\circ}$, and the temperature has the maximal value at $\theta=0^{\circ}$ on the downstream pipe. In comparison with the reference solution ${ }^{(16)}$, it is confirmed that the present approach gives the appropriate solution quantitatively.

\section{Simulation of Counter-flow Heat Exchanger Model}

\subsection{Computational conditions}

Two counter-flow heat exchanger models are considered. One is constructed by the square ducts. Another is the ducts with oblique wavy walls. The basic unit is shown in Fig.17. The oblique wavy wall is defined by

$$
z_{\text {top }}=z_{\text {bottom }}=-A \cos \frac{2 \pi}{L_{x}}(x+y \tan \gamma),
$$

where $A$ is the amplitude of wavy wall and $\chi\left(=\tan ^{-1}\left(L_{x} / D\right)\right)$ is the angle for the $y$ axis. The computational domain is constructed by $3 \times 3$ arrangement of basic unit. The heated air and cooling air are placed alternately with counter-flow. The heat exchange is carried out through wall surface with the infinitesimal thickness. The initial state is the uniform flow with $u=1.0, T=1.0$ for the heated air and $u=-1.0, T=0.0$ for the cooling air. The velocity and temperature are fixed by the uniform flow on the inflow boundary and extrapolated from the inner points on the outflow boundary. On the most outside walls, the non-slip condition for the velocity and the adiabatic condition for the temperature are imposed. For the boundary conditions on the infinitesimal wall, the same manner as the non-slip velocity, the pressure Neumann condition obtained by the normal momentum equation, and the 


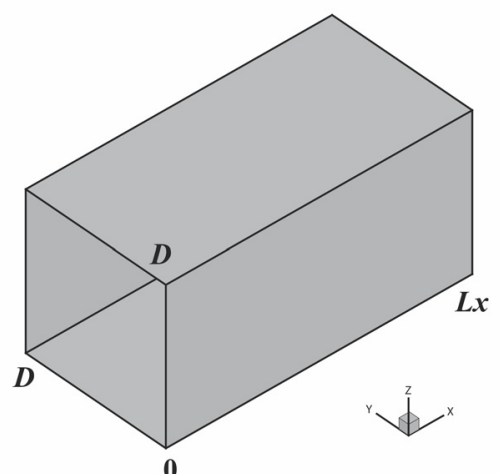

(a) Straight wall.

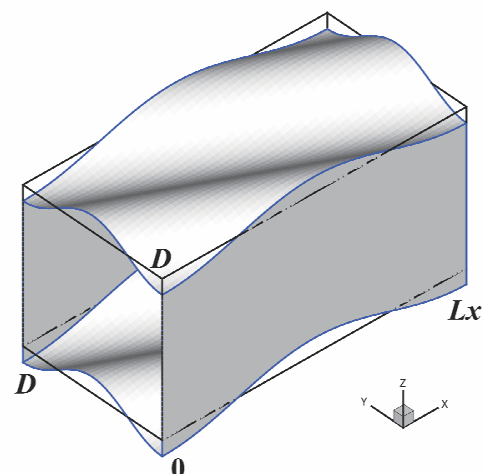

(b) Oblique wavy wall.

Fig. 17 Surface geometries of basic unit.

continuity condition of heat flux, Eq.(5), can be applied. The hydraulic diameter $D_{h}$, the Reynolds number based on $D_{h}$, and the Fanning's friction factor $f$ are defined by

$$
D_{h}=\frac{4 V}{S_{\text {total }}}, R e_{D_{h}}=\frac{U_{b} D_{h}}{v}, f=\frac{(\partial p / \partial x) D_{h}}{2 \rho U_{b}^{2}},
$$

where $V$ and $S_{\text {total }}$ are the volume and surface area of the flow field. The Reynolds number and the Prandtl number are $R e_{D h}=200$ and $P r=0.71$ in this simulation.

\subsection{Simulation of heat exchanger model with straight walls}

In this case, $L x=10.0 D$ and $322 \times 98 \times 98$ grid points are used. Figure 18 shows the arrangement of basic unit. The temperature distribution on $x=5.0 \mathrm{D}$ plane is shown in Fig.19. It is observed clearly that the temperature gradient in each passage is steep near the wall. Then, the heat exchange is done in the wall vicinity. Table 3 shows the comparison of

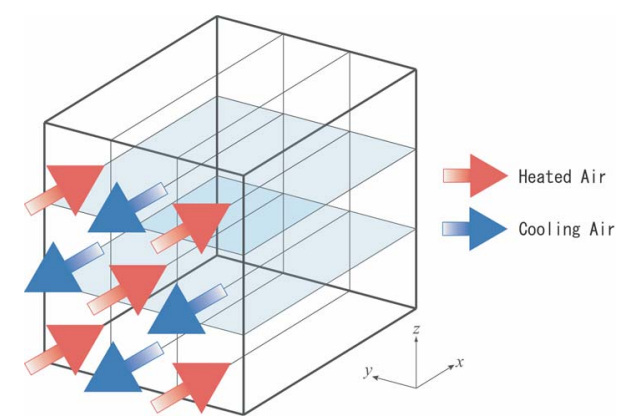

Table 3 Comparison of characteristic quantities.
\begin{tabular}{|l|c|c|c|}
\hline & $f R e_{D h}$ & $u_{\max } / u_{m}$ & $N u$ \\
\hline Present & 15.0 & 2.01 & 3.02 \\
\hline Shah et al. ${ }^{(17)}$ & 14.2 & - & 3.09 \\
\hline Lundgren et al. $^{(18)}$ & - & 2.09 & - \\
\hline
\end{tabular}

Fig. 18 Arrangement of straight basic unit.

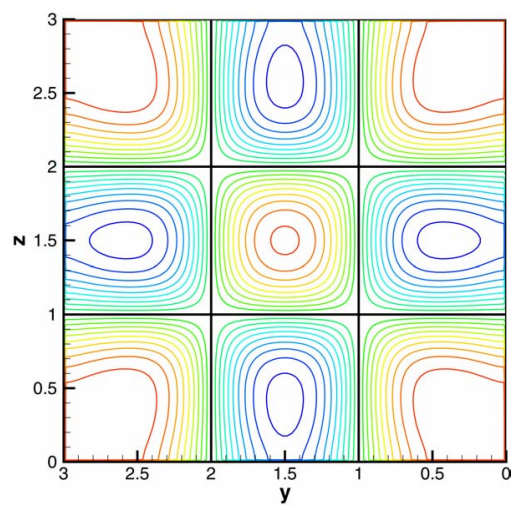

Fig. 19 Temperature contours on $x=5.0 D$. 
characteristic quantities, i.e., the pressure loss $f R e_{D h}$, the ratio between maximum and mean velocities $u_{\text {max }} / u_{m}$, and the Nusselt number $N u$. These physical quantities obtained by the present approach are in good agreement with the reference solutions ${ }^{(17),(18)}$.

\subsection{Simulation of heat exchanger model with oblique wavy walls}

The parameters of oblique wavy wall are $A=0.1 D, \gamma=60^{\circ}, L x=2.0 D$. The arrangement of basic unit is shown in Fig.20. The passage is constructed by 5 basic units and the passage length is $10.0 D$. The number of grid points is $322 \times 98 \times 144$. Figure 21 shows the velocity vectors and streamwise velocity contours of central passage on $x=5.7 \mathrm{D}$ plane. Morimoto et al. ${ }^{(19)}$ reported that the strong rotating flow to the low pressure region was induced, since the flow along the oblique wavy walls was restricted in the side walls. As a result, the clockwise vortex in the upper wall and the counterclockwise vortex in the lower wall were formed. The similar flow pattern is observed clearly in the present solution. Table 4 shows the pressure loss and the Nusselt number of straight and oblique wavy walls and the ratio. In comparison of the oblique wavy wall with the straight wall, the pressure loss becomes larger. However, it is confirmed that the heat transfer is promoted in the oblique wavy wall, because the Nusselt number becomes larger than the case with the straight wall. This is due to the secondary flow induced by the dissimilarity of the oblique wavy walls. Then, for the heat exchanger model, it is found that the efficiency of heat transfer is improved by using the oblique wavy walls. The computational conditions are slight different from the reference simulation $^{(19)}$, so that the present result agrees qualitatively with the reference result.

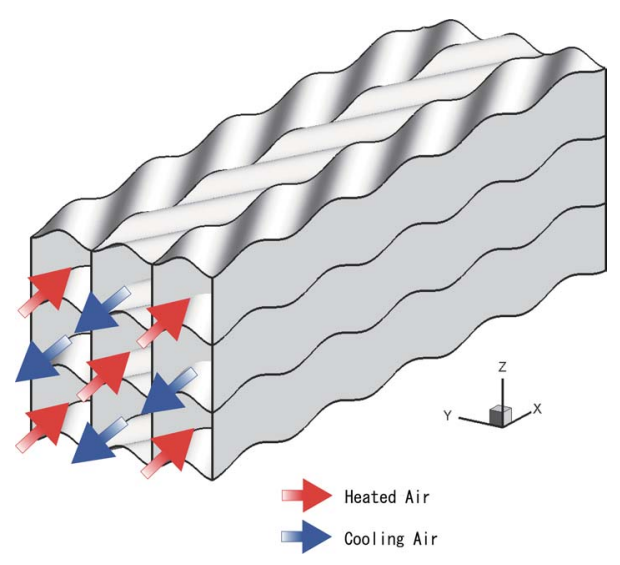

Fig. 20 Arrangement of oblique wavy basic unit.

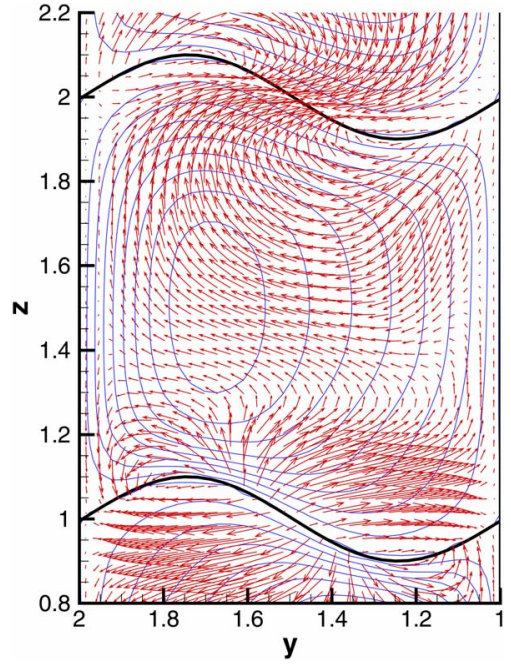

Fig. 21 Velocity vectors and streamwise velocity contours on $x=5.7 D$.

Table 4 Comparison of pressure loss and Nusselt number.

\begin{tabular}{|l|c|c|c|c|c|c|}
\hline \multirow{2}{*}{} & \multicolumn{3}{|c|}{$f R e_{D h}$} & \multicolumn{3}{c|}{$N u$} \\
\cline { 2 - 7 } & Straight & $\begin{array}{c}\text { Oblique } \\
\text { wavy }\end{array}$ & ratio & Straight & $\begin{array}{c}\text { Oblique } \\
\text { wavy }\end{array}$ & ratio \\
\hline Present & 15.0 & 19.47 & 1.298 & 3.02 & 4.30 & 1.42 \\
\hline Morimoto et al. $^{(19)}$ & - & - & 1.430 & - & - & 1.80 \\
\hline
\end{tabular}

\section{Conclusions}

In this paper, the Cartesian grid approach with virtual flux method based on the usual incompressible Navier-Stokes equations is proposed for simulating the incompressible 
multiple flows with heat transfer. First, the present method is validated in flows around a circular cylinder and a pair of circular cylinders in transverse arrangement. As a result, this approach gives the good flow fields quantitatively in comparison with the other results. Then, the method is applied to the consolidated simulations of multiple flows with heat transfer between the heated and cooling flows. In the simulation of heated flows in pipes and cooling flow around pipes, the present approach shows the good agreement with the reference solution. Finally, the simulation of counter-flow heat exchanger model with straight and oblique wavy walls is carried out. The appropriate solution can be obtained in comparison with the reference result. Therefore, it is concluded that the present approach is very fruitful for the consolidated simulation of incompressible complicated multiple flows with heat transfer.

\section{References}

(1) E.A. Fadlun, R. Verzicco, P. Orlandi, and J. Mohd-Yosof, J. Comput. Phys., 161, (2000), pp.35-60.

(2) D. Goldstein, R. Handler, and L. Sirovich, J. Comput. Phys., 105, (1993), pp.354-366.

(3) H. Nishida, Notes on Numerical Fluid Mechanics (Springer), 78, (2001), pp.17-32.

(4) E.M. Saiki and S. Biringen, J. Comput. Phys., 123, (1996), pp.450-465.

(5) I. Tanno, K. Morinishi, K. Matsuno, and H. Nishida, Trans. JSME Ser. B, 70-699, (2004), pp.2689-2696. (in Japanese)

(6) I. Tanno, K. Morinishi, K. Matsuno, and H. Nishida, Trans. JSME Ser. B, 72-714, (2006), pp.217-224. (in Japanese)

(7) Y. Morinishi, T.S. Lund, O.V. Vasilyev, and P. Moin, J. Comput. Phys., 143, 90(1998), pp.90-124.

(8) C.H. Rhie and W.L. Chow, AIAA Journal, 21-11, (1983), pp.1525-1532.

(9) K. Kawakami, H. Nishida, and N. Satofuka, Trans. JSME Ser. B, 60-574, (1994), pp.1891-1896. (in Japanese)

(10) M. Rosenfeld, Computers \& Fluids, 23-5, (1994), pp.693-709.

(11) S.E. Rogers and D. Kwak, AIAA Journal, 28-2, (1990), pp.253-262.

(12) G. Xi, K. Torikoshi, K. Kawabata, and K. Suzuki, Trans. JSME Ser. B, 61-585, (1995), pp.1796-1803. (in Japanese)

(13) E.R.G. Eckert and E. Soehngen, Trans. ASME, 74, (1952), pp.343-347.

(14) B.A./K Abu-Hijleh, Trans. ASME, J. Heat Transfer, 125, (2003), pp.804-811.

(15) K.S. Chang and C.J. Song, Int. J. Numer. Meth. Fluids, 11, (1990), pp.317-329.

(16) M. Shirazaki, M. Iwata, and R. Himeno, Nagare, 22, (2003), pp.247-259. (in Japanese)

(17) R.K.Shah and A.L. London, Dept. Mech. Eng., Stanford Univ., TR75, (1971).

(18) T.S. Lundgren, E.M. Sparrow, and J.B. Starr, J. Basic Eng., Res.9, 2, (1965), pp.207-212.

(19) K. Morimoto, Y. Suzuki, and N. Kasagi, Trans. JSME Ser. B, 70-698, (2004), pp.2604-2611. (in Japanese) 